C. Gaich Shareholder of: Eli Lilly and Company, Employee of: Eli Lilly and Company, A. DeLozier Shareholder of: Eli Lilly and Company, Employee of: Eli Lilly and Company, A. Quebe Shareholder of: Eli Lilly and Company, Employee of: Eli Lilly and Company, L. Sun Shareholder of: Eli Lilly and Company, Employee of: Eli Lilly and Company, S. Otawa Employee of: Eli Lilly and Company, J. Pope Grant/research support from: Amgen, Bayer, BMS, GSK, Merck, Novartis, Pfizer, Roche, UCB, Consultant for: AbbVie, Amgen, Bayer, BMS, Celtrion, Lilly, Merck, Novartis, Pfizer, Roche, UCB

DOI: 10.1136/annrheumdis-2018-eular.2001

\section{FRI0014 LOW-DOSE ASPIRIN MAY HAVE A ROLE AS PRIMARY PROPHYLAXIS OF CARDIOVASCULAR EVENTS IN RHEUMATOID ARTHRITIS: EVIDENCE FROM AN ITALIAN MULTICENTRIC RETROSPECTIVE STUDY}

D. Iacono $^{1}$, S. Fasano' ${ }^{1}$, I. Pantano ${ }^{1}$, V. D'Abrosca' ${ }^{1}$, P. Ruscitti ${ }^{2}$, D.P.E. Margiotta ${ }^{3}$ L. Navarini ${ }^{3}$, N. Maruotti ${ }^{4}$, F.P. Cantatore ${ }^{4}$, A. Afeltra ${ }^{3}$, R. Giacomelli ${ }^{2}$, G. Valentini ${ }^{1}$ ${ }^{1}$ Rheumatology, University of Campania Luigi Vanvitelli, Naples; ${ }^{2}$ Rheumatology, University of L'Aquila, L'Aquila; ${ }^{3}$ Rheumatology, Campus Bio-Medico, Rome; ${ }^{4}$ Rheumatology, University of Foggia, Foggia, Italy

Background: Cardiovascular (CV) morbidity and mortality are significantly greater in Rheumatoid Arthritis (RA) patients than in the general population. Acetylsalicylic acid (ASA) is known to be associated with a significant decrease in the incidence of $\mathrm{CV}$ events in patients at high $\mathrm{CV}$ risk, as we have recently demonstrated in patients with Systemic Lupus Erythematosus, but its effectiveness as primary prophylaxis in RA patients has not yet been addressed.

Objectives: To investigate the role of ASA in reducing the incidence of CV events in an Italian multicentre RA cohort from the GIRRCS (Gruppo Italiano di Ricerca in Reumatologia Clinica e Sperimentale).

Methods: The clinical charts of RA patients consecutively admitted to 4 GIRRCS centres for their 1 st visit from November 1 st 2000 to December 31 st 2015, who, at admission, satisfied 2010 ACR/EULAR criteria for RA and had not experienced any CV event, were analysed. The incidence of CV events during follow-up was recorded at December 2016. Kaplan Meier curve and log-rank test were used to investigate differences in event-free survival. Cox regression analysis served to identify factors associated with CV event occurrence.

Results: Seven hundred and forty-six consecutive RA patients were enrolled and followed up for a median of 5.6 years (range 2.9-8.9 years). The incidence rate (IR) of CV events was 7.8/1000 person-years (pys) in the overall cohort. Patients were subdivided into two groups, namely ASA- (242 patients) and non-ASAtreated (504 patients). The IR of CV events was significantly lower in the ASAtreated with respect to the non ASA-treated group (IR 1.7 vs 11.5/1000 pys; $\mathrm{p}=0.0002)$. Furthermore, the $\mathrm{CV}$ event-free rate was longer in ASA-treated than in non-ASA-treated patients (log-rank test 12.3; $\mathrm{p}=0.0004)$. Figure 1.

At multivariate analysis hypertension and metabolic syndrome (HR 5.6, 95\% Cl:1.2-26.3;p 0.03 and $\mathrm{HR} 3.7,95 \% \mathrm{Cl}: 1.3-9.8 ; \mathrm{p} 0.009)$ resulted to be the only positive predictors; ASA treatment (HR 0.04, 95\% Cl:0.06-0.33;p 0.02) the only negative one.

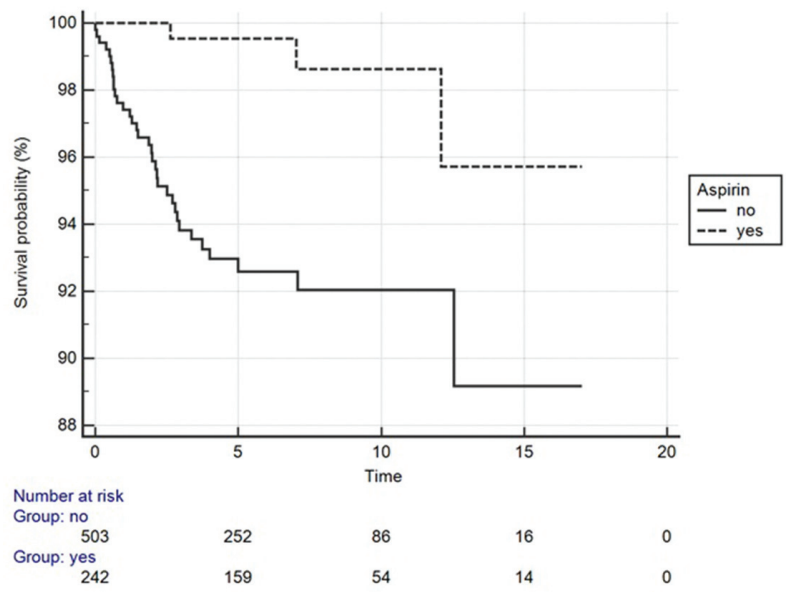

Conclusions: The incidence rate of CV events in our italian multicentric cohort was lower than that reported in other European and non European cohorts. Lowdose ASA may have a role in the primary prophylaxis of CV events in RA patients.

\section{REFERENCES:}

[1] del Rincón ID, et al. High incidence of cardiovascular events in a rheumatoid arthritis cohort not explained by traditional cardiac risk factors. Arthritis Rheum 2001;44(12):2737-45.

[2] Fasano S, et al. Longterm Hydroxychloroquine Therapy and Low-dose Aspirin May Have an Additive Effectiveness in the Primary Prevention of Cardiovascular Events in Patients with Systemic Lupus Erythematosus. J Rheumatol 2017;44(7):1032-1038.

[3] Meek IL, et al. Cardiovascular case fatality in rheumatoid arthritis is decreasing; first prospective analysis of a current low disease activity rheumatoid arthritis cohort and review of the literature. BMC Musculoskelet Disord 2014;15:142

Disclosure of Interest: None declared

DOI: 10.1136/annrheumdis-2018-eular.1649

\section{FRI0015 TREATING RHEUMATOID ARTHRITIS TO TARGET: IS LOW DISEASE ACTIVITY GOOD ENOUGH?}

E. Nikiphorou ${ }^{1}$, S. Norton ${ }^{1}$, A. Young ${ }^{2}$, L. Carpenter ${ }^{1}$, J. Dixey ${ }^{3}$, D. Walsh ${ }^{4}$, P. Kiely ${ }^{5}$, on behalf of ERAS-ERAN working group. ${ }^{1}$ Academic Rheumatology, King's College London, London; ${ }^{2}$ Life Sciences, University of Hertfordshire, Hertfordshire; ${ }^{3}$ Rheumatology, The Royal Wolverhampton, Wolverhampton; ${ }^{4}$ Rheumatology, The University of Nottingham, Nottingham; ${ }^{5}$ Rheumatology, St George's Hospital, London, UK

Background: Treat-to-target (T2T) principles in rheumatoid arthritis (RA) are now widely recognised as effective in achieving optimal disease outcomes.

Objectives: To examine for differences in outcomes between low (LDAS) and remission (RDAS) disease activity score (DAS) categories, addressing the question: is LDAS a 'good enough' treatment target in RA?

Methods: Data from two consecutive UK multi-centre RA inception cohorts with similar design were used: the Early RA Study (ERAS) and Early RA Network (ERAN). Recruitment figures/median follow up for ERAS and ERAN were 1465/ 10 years (maximum 25 years), and 1236/6 years (maximum 10 years) respectively. Standard demographic and clinical variables were recorded at baseline and then annually until the end of study follow up. Disease activity was categorised by mean DAS28 score between years $1-5$ as remission [mRDAS $<2.6$ ] or low [mLDAS 2.6-3.2]; as sustained low/remission DAS (sLDAS/sRDAS) based on DAS persisting in each of the two categories at years 1-2 and as Boolean remission (years 1-2). Change in HAQ and SF36 (physical [PCS] and mental [MCS components) for each disease activity category were modelled using linear mixed models with time incorporated as a linear spline with change-point at 12 months. Year of onset, age, gender and use of steroids or conventional DMARDS at first visit were included as covariates.

Results: Out of 2701 patients, 468 (17\%) were in mRDAS, $284(11 \%)$ in mLDAS in the first five years of disease. Lower proportions had achieved SRDAS (8\%), sLDAS (6\%) and Boolean (2\%) remission. Mean age was similar across categories; more women were in low vs remission DAS. Compared to mLDAS or sLDAS, inflammatory markers, DAS, functional (HAQ, PCS) and mental (MCS) scores tended to be better in the mRDAs, sRDAS or Boolean remission categories (figure 1). Significant differences $(p<0.05)$ were noted between the mRDAS and mLDAS between years 1-5 for all outcomes; for sRDAS compared to sLDAS, the difference was significant at one year but not by five years.

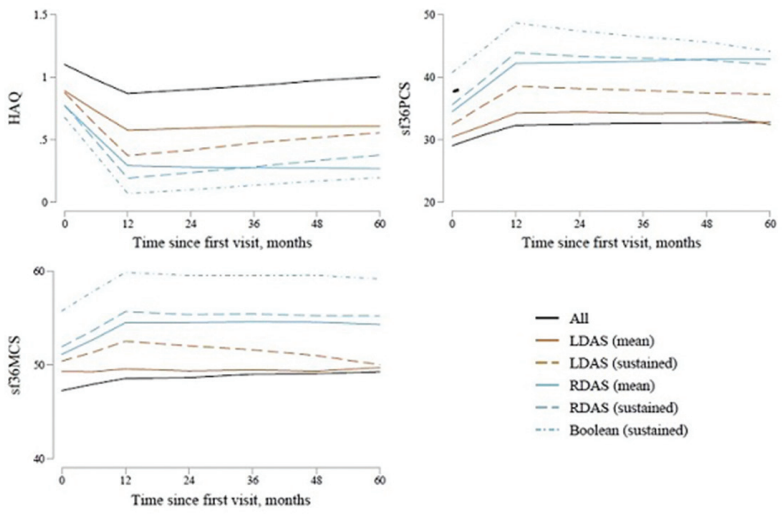

Conclusions: The results demonstrate striking differences between remission and low DAS categories, suggesting worse functional and SF36 outcomes ove 
time in the low DAS categories. This supports that remission should be the primary T2T goal in in RA.

Disclosure of Interest: None declared

DOI: 10.1136/annrheumdis-2018-eular.3890

\section{FRI0016 NO RELATIONSHIPS BETWEEN ACPA AND PERIODONTITIS IN EARLY RHEUMATOID ARTHRITIS}

F. Mechid $^{1}$, C. Zehraoui ${ }^{1}$, M. Meddad ${ }^{2}$, S. Salah ${ }^{3}$, M. Benidir ${ }^{3}$, S. Merad ${ }^{4}$, N. Saidi ${ }^{1}$, O. Cheikh ${ }^{1}$, N. blidi ${ }^{1}$, C. Dahou ${ }^{1}$. ${ }^{1}$ Rheumatology, Mohamed Lamine Debaghine hospital; ${ }^{2}$ Periodontology, Mustapha Bacha Hospital; ${ }^{3}$ Immunology; ${ }^{4}$ Bacteriology, Dely brahim, institut Pasteur, Algiers, Algeria

Background: Proteins citrullination contributes to generate anticitrullinated peptide antibodies (ACPA) in rheumatoid arthritis (RA). Porphyromonas gingivalis $(\mathrm{Pg})$ is one of main germs incriminated in the development of periodontitis (PD), it has an enzyme called peptidyl arginine deiminase which is able to citrullinate the host proteins

Objectives: The aim of this study was to seek for a possible association between ACPA and periodontitis

Methods: We conducted a case-control study of 69 patients with early rheumatoid arthritis ( $\leq 2$ years), naive of biotherapy and 138 age-and sex matched healthy controls. Smokers, diabetics, and subjects who received dental care and those who used antibiotics in the previous 6 months were not included. Demographic data and ACPA were determined. A periodontal examination was performed to all participants. Subgingival plaque samples were analysed to seek for Porphyromonas gingivalis $(\mathrm{Pg})$ in both population in the case of periodontitis.

Results: The mean age of our patients was $40.75 \pm 12.04$, the mean duration of the illness was $14.30 \pm 6.76$ months (extremes: 1-24 months). ACPA was detected in $88 \%$ of patients and the mean titre was $255.57 \pm 409.78$. PD frequency was higher in patient with PR compared with healthy controls (43\% versus $29 \%$ ) and a significant association was found between PR and PD $(p<10-3)$. Patients with RA had $2.46(\mathrm{Cl} 1.12$ to 5.39$)$ higher odds of having PD compared with healthy controls. In early RA, ACPA titre and rate was not associated with PD $(p=0.06, p=0.44$ respectively). Regarding the frequency of Porphyromonas gingivalis, there was no significant difference between the PR group and the control group $(p=0.45)$. In addition, there was no significant difference between RA group and controls $(p=0.68)$ concerning Porphyromonas gingivalis and ACPA

Conclusions: Periodontitis is a risk factor for the occurrence of rheumatoid arthritis. The ACPA does not seem to be related to periodontitis. In addition there was no association between ACPA and the presence of porphyromonas gingivalis.

\section{REFERENCES:}

[1] Eriksson K, Nise L, Kats A, Luttropp E, Catrina Al, Askling J, Jansson L, Alfredsson L, Klareskog L, Lundberg K, Yucel-Lindberg T. Prevalence of Periodontitis in Patients with Established Rheumatoid Arthritis: A Swedish Population Based Case-Control Study. PLoS One 2016;11(5):e0155956.

[2] Laugisch O, Wong A, Sroka A, Kantyka T, Koziel J, Neuhaus K, Sculean A, Venables PJ, Potempa J, Möller B, Eick S. Citrullination in the periodontium -a possible link between periodontitis and rheumatoid arthritis. Clin Oral Investig 2016;20(4):675-683.

[3] Dissick A, Redman RS, Jones M, Rangan BV, Reimold A, Griffiths GR, et al. Association of Periodontitis with Rheumatoid Arthritis: A Pilot Study. Journal of Periodontology 2010;81:223-230.

[4] Mikuls TR, Payne JB, Yu F, et al. Periodontitis and porphyromonas gingivalis in patients with rheumatoid arthritis. Arthritis \& Rheumatology 2014;66(5):1090-100.

Disclosure of Interest: None declared

DOI: 10.1136/annrheumdis-2018-eular.5346

\section{FRI0017 AN EXPLORATORY STUDY ON THE ROLE OF VITAMIN D SUPPLEMENTATION IN IMPROVING PAIN AND DISEASE ACTIVITY IN RHEUMATOID ARTHRITIS}

G. Adami ${ }^{1}$, O. Viapiana ${ }^{1}$, L. Bogliolo ${ }^{2}$, F.P. Cantatore $^{3}$, M. Varenna ${ }^{4}$, N. Malavolta ${ }^{5}$, A. Del Puente ${ }^{6}$, M. Muratore ${ }^{7}$, G. Orsolini ${ }^{1}$, D. Gatti ${ }^{1}$, M. Rossini ${ }^{1}$, on behalf of Study Group on Osteoporosis and Metabolic Skeletal Diseases of the Italian Society of Rheumatology (SIR). ${ }^{1}$ Rheumatology Unit, University of Verona, Verona; ${ }^{2}$ Rheumatology Unit, Policlinico San Matteo Foundation/University of Pavia, Pavia; ${ }^{3}$ Rheumatology Unit, University of Foggia, Foggia; ${ }^{4}$ Rheumatology Unit, Gaetano Pini Institute, Milano; ${ }^{5}$ Rheumatology Unit, University of Bologna Sant'OrsolaMalpighi Hospital, Bologna; ${ }^{6}$ Rheumatology Unit, University Federico II of Naples, Napoli; ${ }^{7}$ Rheumatology Unit, Galateo San Cesario Hospital, Lecce, Italy

Background: Lower serum vitamin D levels have been associated with various autoimmune disorders. Especially in patients affected by Rheumatoid Arthritis
(RA), has been observed an inverse correlation between serum levels of 25hydroxyvitamin D (25OHD), pain and disease activity, but the cause-effect relationship is not clear

Objectives: The aim of this exploratory study is to investigate the effects of supplementation with cholecalciferol (vitD3) in improving pain and disease activity in RA patients with or without vitamin D deficiency $(250 \mathrm{HD}<20 \mathrm{ng} / \mathrm{ml})$.

Methods: In this prospective open-label intervention study, patients fulfilling the EULAR/ACR 2010 criteria for diagnosis of RA, in non-remission (DAS28$\mathrm{CRP}>2,6$ ), on stable disease-modifying antirheumatic drugs, and whose treatment was not expected to be changed over a 3 month period following inclusion, were recruited. DAS28-CRP, VAS pain and serum levels of $250 \mathrm{HD}$, were evaluated at the baseline and after 3 months of supplementation with oral 100,000 IU/ monthly of vitD3.

Results: A sample composed by 61 patients (47 females), with an average age (SD) of 5812 years within $26-86$ years range were included. At baseline the mean (SD) $25 \mathrm{OHD}$ levels were $2210 \mathrm{ng} / \mathrm{mL}$. $57 \%$ of the patients were found to have vitamin D deficiency $(<20 \mathrm{ng} / \mathrm{mL}$ ). Mean serum $250 \mathrm{HD}$ levels improved from $13 \pm 5$ to $32 \pm 12$ and from $29 \pm 7$ to $41 \pm 10 \mathrm{ng} / \mathrm{mL}$ in patients with or without vitamin $D$ deficiency, respectively. At baseline, mean VAS pain was significantly higher in patients with vitamin D deficiency. In the figure are shown DAS28-CRP and VAS pain at baseline and after 3 months of vitD3 supplementation both in patients with or without vitamin D deficiency. After large doses of VitD3, VAS pain significantly decreased in patients with vitamin D deficiency, while DAS28-CRP significantly improved only in patients without vitamin $D$ deficiency at baseline.

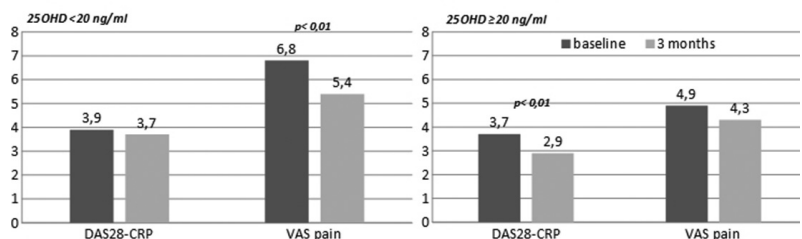

Conclusions: VitD3 supplementation appears to be associated with significant and different effects on pain and disease activity in RA patients dependent on $250 H D$ serum levels. Vitamin D deficiency $(<20 \mathrm{ng} / \mathrm{mL})$ seems to be mainly correlated with pain, while higher levels of $25 \mathrm{OHD}$ might have immunomodulatory effects. A randomised, double-blind, low versus high vitD3 dose, placebo-controlled trial is recommended.

Disclosure of Interest: None declared

DOI: 10.1136/annrheumdis-2018-eular.5235

\section{FRI0018 THE ABILITY OF DISEASE ACTIVITY MEASURES TO PREDICT MAJOR THERAPEUTIC CHANGE IN US VETERANS WITH RHEUMATOID ARTHRITIS}

G. Cannon ${ }^{1}$, C.-C. Teng ${ }^{1}$, N.A. Accortt ${ }^{2}$, D.H. Collier ${ }^{2}$, T.-C. Lin ${ }^{2}$, B.C. Sauer ${ }^{1} .{ }^{1}$ Salt Lake City VA Medical Center and University of Utah, Salt Lake City; ${ }^{2}$ Amgen, Thousand Oaks, USA

Background: Current rheumatoid arthritis (RA) treatment guidelines recommend the use of disease activity measures (DAMs) to guide RA therapy. These guide lines recommend considering escalation of therapy in RA patients with high or moderate disease activity. Recent work by our group has demonstrated that many RA patients with high/moderate RA by Disease Activity Score with 28 joints (DAS28) did not have therapy escalated despite active disease (DAS28 $\geq 3.2$ ).

Objectives: 1) To determine if the rate of major therapeutic change (MTC) for RA patients with high/moderate disease activity based on DAS28 was similar when measured using two other common DAMs; 2) to compare the ability of different DAMs to predict MTC across the full spectrum of RA disease activity.

Methods: US Veterans enrolled in the VA Rheumatoid Arthritis (VARA) registry with 1) a complete set of DAMs (DAS28, Clinical Disease Activity Index [CDAI], Routine Assessment of Patient Index Data 3 [RAPID3]) recorded (index date), 2) two other visits during the preceding 18 months separated by at least 60 days, and 3) clinical data available for 18 months prior to through 30 days following index date were eligible. Each patient was assessed for MTC within 1 week before and 30 days after index date. MTC was defined as any of the following: 1) initiation of new biologic or nonbiologic DMARD, 2) escalation of DMARD dose by $\geq 25 \%$, 3) initiation of prednisone (as new agent or after 90 day gap during baseline), or 4) increase in monthly average prednisone dose by $25 \%$ and/or 5 ) injection of 2 or more joints with corticosteroids. MTC was analysed by DAM severity thresholds of 1) high, moderate, low, and remission, and 2) high, high/moderate, and high/moderate/low levels. Analyses of the latter thresholds included sensitivity, specificity, predictive values, and accuracy estimations for MTC at each DAM level.

Results: Of 1776 eligible patients, $89 \%$ were male, mean age was 63.4 years, mean disease duration was 13.4 years, $79 \%$ tested positive for rheumatoid factor, 